\title{
Accretion Disks and Line Emission in AGN
}

\author{
E. Rokaki ${ }^{1}$
}

Igglesi 2, N. Kosmos, 11743 Athens, Greece

\begin{abstract}
Irradiated accretion disks around massive black holes are expected to produce part of the line spectrum of AGN, but most of the disk emission must be thermal, observed at UV wavelengths. The two emission components, lines and continuum, are fitted by a unique accretion-disk model that gives the mass of the black hole and the inclination of the disk. The distribution of the disk inclination in a complete sample of Seyfert 1 galaxies suggests that their nuclei are orientation-selected, affected by strong absorption at low disk latitudes. The black-hole masses in the same sample confirm the long-standing non-linearity between $M$ and $L$ for AGN and the non-causal relationship between nearby Seyfert 1 galaxies and distant quasars (i.e., pure luminosity evolution is ruled out).

Irradiated accretion disks are also combined with the relativistic jet model in order to constrain the orientation and the Lorentz factor of 14 superluminal radio sources. At least for a few objects, the line and the radio data are inconsistent with both models, unless a new parameter (jet bending, a second emission-line component, etc.), is also involved. Despite this inconsistency and the ambiguous evidence for combined disk and jet fits in the remaining superluminal sources, a successful merger of these two models might address questions about the nature of AGN and also constrain the Hubble constant.
\end{abstract}

\section{Introduction}

As a challenge to theorists, the accretion-disk model of the AGN has proven to be particularly tricky. Nevertheless, on account of its predictive nature, disk theory provides many opportunities for comparing theory and observations and gives us hope for understanding these objects. Below I outline my personal view of the disk model, integrated in our general beliefs of AGN structure. If the disk model proves to be a good fit to the general picture of AGN, it can shed light on the black-hole question, on AGN physics, on AGN geometry and obscuration, and on the Hubble constant.

Accretion disks provide the most exciting explanation of the double-peaked, blue-boosted, and redshifted broad-line profiles that are observed in a few AGN sources, because they give not only the orientation of the disk (Chen et al. 1989), but also the mass of the central black hole (Rokaki et al. 1992). Obviously, an

\footnotetext{
${ }^{1}$ On leave from Royal Observatory of Edinburgh.
} 
unambigous disk line profile would be direct observational evidence of massive black holes in AGN.

In contrast to the thermal accretion-disk model (Lynden-Bell 1969), the line model (Shields 1977) has been pursued only in a the case of a few AGNs which have double-peaked emission-line profiles. The fact that the model predicts a diversity of line profile shapes (Dumont \& Collin-Souffrin 1990) enables the investigation of the disk line emission in large AGN samples without any criterion on the shape of the broad emission lines. In the following, I discuss two such studies.

The first study confirms the consistency between the line and the thermal disk model and finds the central mass and orientation of the disk in a complete sample of Seyfert 1 sources. Both line-emitting disk-model and unified-scheme lovers can still be happy: the line and continuum emission of the Seyfert 1 nuclei fit in the same flat structure, and probing the orientation in these sources should reveal high absorption at low disk latitudes.

The second work points out the near-inconsistency between the line-emitting disk model and the relativistic-expansion model (Rees 1968) by searching for face-on disk signatures in the line profiles of superluminal radio sources. There is no strong evidence that the two models should fit together, but there are many unstudied sources in which strong evidence might be found. Superluminal motions and double-peaked line profiles make 3C 390.3 (Pérez et al. 1986) a good candidate. The line and continuum variability of this source (described in several papers in this volume) can be used to search for a causal relationship between different emission components across the electromagnetic spectrum (Rokaki et al. 1993), constrained in orientation and Lorentz factor by radio data.

Since everything in extragalactic astronomy uses the Hubble constant, very high apparent velocities of a source of known geometry will set upper limits on this fundamental parameter. However, this can be expected to be difficult in practice.

\section{The Complete Sample}

Double-peaked line profiles favor the accretion-disk model, but if the disks are seen in double-peaked lines, they should be also observed with a variety of lineprofile shapes. Indeed, models predict this diversity, which can be tested with observed AGN emission lines. The accuracy of the test, as well the significance of the derived physical parameters, is optimized when the sample of the AGN sources is complete. The Piccinoti et al. (1982) sample has 34 AGN sources selected in the hard X-ray sky survey, and it is complete. It has 21 broad-line emission sources, which also form a complete sample.

Optical and UV spectra of this sample (simultaneous for one third of the sources) were collected in order to test the line-emitting disk model of CollinSouffrin \& Dumont (1989) and the thermal-disk model of Cunningham (1975). Both models have relativistic corrections and the disk continuum spectrum (multi-temperature blackbody emission) is calculated at two extreme values of the black hole spin. The black-hole rotation is rather unimportant for the line emission, which comes from large disk radii $\left(>100 R_{S}\right)$. 


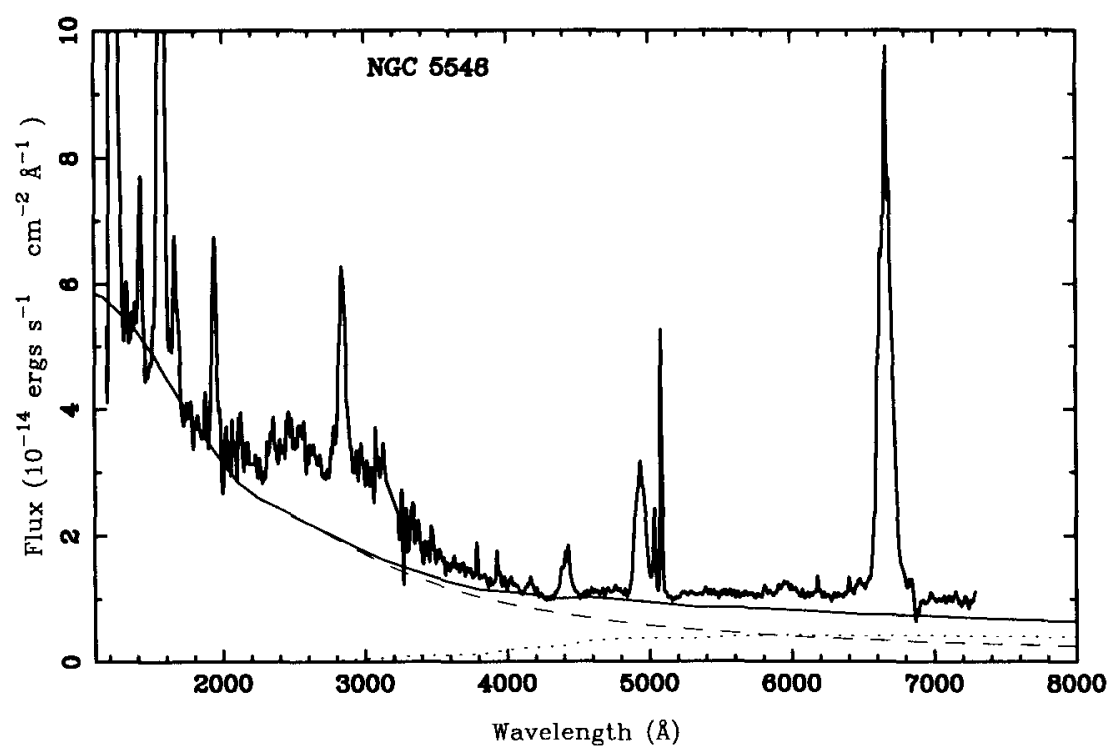

Figure 1. A fit (solid line) of the optical to the UV continuum of NGC 5548 which sums a disk (dashed line) and a stellar component (dotted line). Physical parameters are given in the text.

The broad $\mathrm{H} \beta$ emission line and the UV continuum shape were fitted independently without any preconceptions about orientation; the inclination is constrained only to very low angles from the line profile. Note that since the probability of observing a disk geometry is proportional to the sine of the inclination, the disk model predicts that a complete and a non-orientation-selected sample will have a mean inclination angle close to $60^{\circ}$. Details in this work can be found in Rokaki \& Boisson (1996), and here I outline the method and the major results.

\subsection{Mass and Orientation Measurement}

Figure 1 shows an accretion-disk fit to the UV continuum of NGC 5548. The dashed line is the thermal spectrum of a face-on disk fit with an accretion rate of $0.007 M_{\odot} \mathrm{yr}^{-1}$. The mass of the black hole is $8.3 \times 10^{7} M_{\odot}$ and has the maximum possible spin. An equally good fit can be obtained with a non-rotating black hole, but it must be 2.5 times less massive and an mass-accretion rate that is lower by a factor of 3 on account of its lower efficiency. By increasing the disk inclination, the fits give more massive black holes due to the fact that the high inclinations as the low masses harden the spectrum (see Fig. 7 of Cunningham 1975).

The two solid curves of Fig. 2 give for Kerr and Schwarzschild black holes the mass solutions from fits to the data of Fig. 1. As expected, the mass decreases at low inclinations. Since the points of these curves give identical spectra it is 


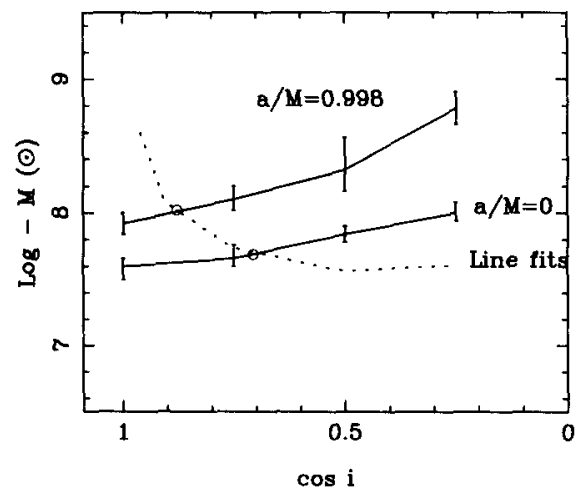

Figure 2. Black-hole mass as a function of $\cos i$ of NGC 5548 as derived from the continuum (solid lines) and line (dashed line) disk fits.

impossible to extract any information about the disk inclination and mass by modeling the UV continuum only. However, these parameters can be determined very precisely if there are additional constraints with a different relationship between the dynamics and the geometry. And indeed this happens in fitting disks to broad-line profiles.

Let $d R$ be a disk ring of infintesimal width at radius $R$ in Keplerian rotation around a mass $M$. The rotation axis and the line of sight make an angle of inclination $i$. If it is supposed that this ring emits constant line flux $F$, and that its emission can be fitted to an observed line profile of $H W Z I=w$ and of total line flux $F_{o b s}$, we get (neglecting relativistic corrections)

$$
w=\sin i\left(G M R^{-1}\right)^{1 / 2}
$$

and

$$
F_{o b s}=f(z, h) F \cos i \int R d R=-2 G^{2} M^{2} f(z, h) F \cos i \sin ^{4} i \int w^{-5} d w .
$$

The above equation is equivalent to

$$
M^{2} \sin ^{4} i \cos i=c(z, h, F, w),
$$

where $f$ and $c$ are functions of known parameters ( $z$ is the redshift of the source and $h$ the Hubble constant in units of $100 \mathrm{~km} \mathrm{~s}^{-1} \mathrm{Mpc}^{-1}$ ). Equation (3) shows that the central mass increases very rapidly at low inclinations. A similar dependence between $M$ and $i$ is found from disk fits to real line profiles (see the dashed line of Fig. 2). Note that the disk does not fit $\mathrm{H} \beta$ when $i<16^{\circ}$, which gives central mass $M=4 \times 10^{8} M_{\odot}$. At lower inclinations, the model asymmetries (see Fig. 3) strengthen, and do not fit the data. The intersection point of the line and continuum curves gives therefore the unique $M$ and $i$ that fit both the line and continuum. The determined values of $M$ and $i$ might depend on the details of the thermal and line-emission model, but the hypothesis of accretion-disk line emission gives a unique solution of the central mass and orientation of the disk. 


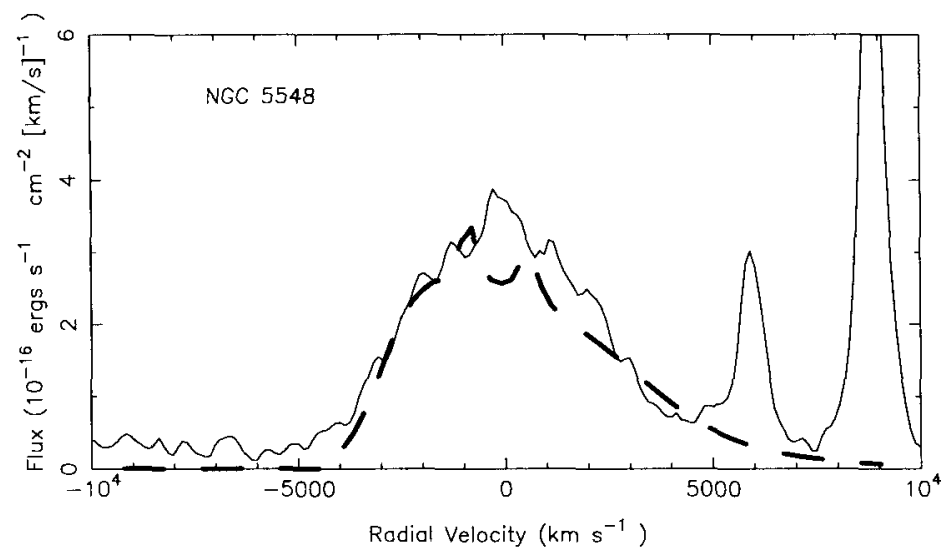

Figure 3. A disk fit (dashed line) to the broad $\mathrm{H} \beta$, line profile of NGC 5548. Narrow lines have not been subtracted from the data. Model parameters are given in the text.

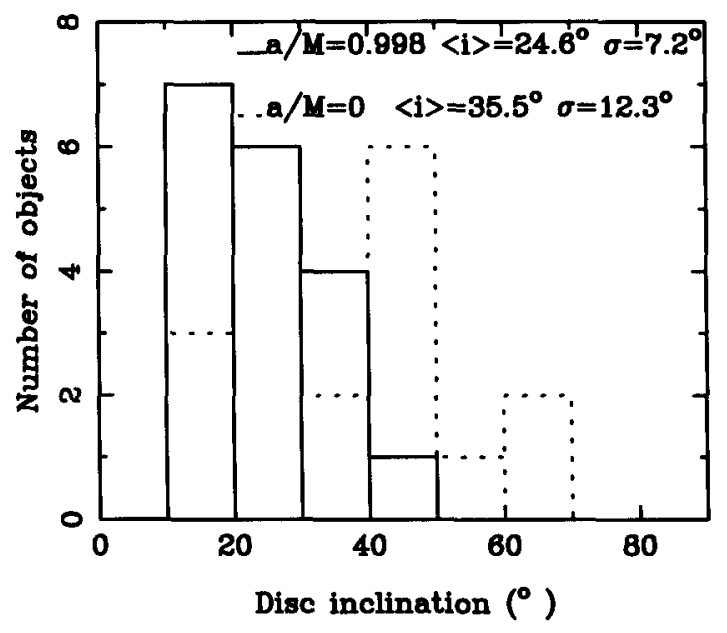

Figure 4. The disk inclination distribution in the Seyfert 1 sources of the Piccinoti et al. sample assuming a Kerr (solid line) and a Schwarzschild (dashed line) metric. 


\subsection{Orientations}

The disk inclinations of the Seyfert 1 nuclei in the Piccinoti et al. sample for the two different metrics assumed are shown in Fig. 4. Note that 3 objects (NGC 3227, MCG-6-30-15, and 3C 345) in the Kerr model and one (NGC 3227 ) in the Schwarzschild model are not included in the present analysis since we find no consistent solution. Absorption could be responsible for this, since UV and optical radiation are affected differently. Obviously, absorption can also affect the disk-inclination distribution and contribute to its low mean value $\left(24^{\circ}\right.$ and $35^{\circ}$, with corresponding average deviations $7^{\circ} .2$ and $12^{\circ} .3$ for the Kerr and Schwarzschild geometries, respectively). Note, however, that no source was found with an inclination lower than $10^{\circ}$; this might be due to the low probability of observing such disks, and/or to a dramatic change of the line/continuum ratio at low inclinations.

The unexpected inclination distribution suggests that a new parameter is involved in this sample. Host-galaxy extinction (which has not been taken account) corresponding to $E(B-V) \approx 0.05$ will move the real disk inclination by about $5^{\circ}$ to lower values. If the host-galaxy absorption is independent of the direction, we would not expect to find any edge-on disks. However, these disks have higher a probability of being observed, and therefore they should be the most numerous in the sample. The fact that the majority of the sources would have been moved to inclinations $i<40^{\circ}$ suggests strong absorption in the host galaxy. If this is true, we should have found more objects without consistent solutions. For these reasons, the results rather suggest that the host absorption is orientation-dependent, stronger at low disk latitudes and possibly from the outer disk.

Seyfert 1 nuclei, independently of the rotation of the central black hole, appear as a population selected with orientation around $30^{\circ}$. The absence of face-on disks is rather puzzling (low probability, non-asymmetric lines in the sample, or beaming, perhaps). However, the disk model predicts that face-on disks have low line/continuum ratios (even without considering a beaming of a non-thermal source) since the consistent solutions in lower inclination demand stronger UV fluxes and fainter emission lines. Radio-quiet sources then might be more suitable for observing the predicted line asymmetries of the face-on disks.

\subsection{Masses}

The derived black-hole masses are significantly, but not linearly, correlated with the disk and the X-ray luminosity, affirming a long-standing result that higherluminosity objects which harbor more massive black holes accrete at higher accretion rates in Eddington units. However, the UV and the X-ray luminositymass dependency are different. Kerr black holes give $L_{\text {disk }} \propto M^{2.05 \pm 0.42}$ and $L_{X} \propto M^{1.65 \pm 0.3}$. A similar slope difference has been found in the Schwarzschild metric, and both are unaffected by X-ray variability. If $L_{X}$ and $L_{d i s k}$ are intrinsically related differently to the black-hole mass, the dominance of the disk luminosity over $L_{X}$ should appear when $M \geq 5 \times 10^{7} M_{\odot}$. However host-galaxy absorption could change this result and could be possibly the only source of the effect in this particular sample.

The differential mass function $\left(\phi(M) \propto M^{-4.5}\right)$ and the total nuclear mass density $\left(\rho \lesssim 6 \times 10^{12} M_{\odot} \mathrm{Gpc}^{-3}\right)$ of black holes in Seyfert 1 nuclei based on the 
results of this sample can be found in Rokaki \& Boisson (1996). The steepness of the former function and the low value of the total integrated mass density of Seyfert 1 nuclei (compared to $\rho>2 \times 10^{14} M_{\odot} \mathrm{Gpc}^{-3}$ estimated for quasars; see Cavalier \& Padovani 1988) confirm the non-continuous luminosity evolution from quasars to Seyfert 1 galaxies.

\section{Superluminal Sources}

The disk inclination determined by a line profile fit can provide potential constraints in the case of a superluminal source of an apparent expansion speed $\beta_{a p p} c$, since the equation

$$
\beta_{a p p}=\frac{\beta \sin i}{1-\beta \cos i}
$$

gives the true speed of the expansion $\beta c$ (supposed in the disk axis). For this reason Rokaki, Economou, \& Lawrence (1996), have obtained H $\alpha$ spectra of 14 superluminal radio sources (see Vermeulen \& Cohen 1994 for a recent list) with $z>0.5$ using CGS4. Strong line emission was detected in all but 3 sources. Disk fits to the observed $\mathrm{H} \alpha$ lines are achieved in most of the sources, but the disk inclination has large uncertainties. However, the inclination can be further constrained by requiring consistency of $i$ with the measured expansion speed. Since a lower limit of the inclination is imposed from the absence of strong line asymmetries, this consistency might be not always possible. This is the case for $\mathrm{F} 1308+326\left(\beta_{a p p}=20.8 h^{-1}\right)$ in which the disk fit gives $i>12^{\circ}$. Jet bending, a second line-emission component, and/or large uncertainties in the radio data could cause this inconsistency and obviously they will complicate the interpretation of line and radio data in these sources. However, a future strong evidence of accretion-disk line emission in a superluminal source can constrain the Lorentz factor in that source and thus possibly provide an upper limit on the Hubble constant.

\section{Conclusions}

The accretion-disk model, despite early and persistent problems, remains a powerful theoretical tool for probing AGN physics (even if disks exist only in a small fraction of AGN sources) because it makes observationally testable predictions about the nature of the AGN sources, the evolution of GN (if most of AGN have accretion disks), and the Hubble constant (from future strong evidence for an accretion disk in a source of high superluminal speed).

Acknowledgments. I thank Suzy Collin, Catherine Boisson, Frossie Economou, Andy Lawrence, IAU, the Chinese Academy of Sciences, and Brad Peterson.

\section{References}

Cavalier, A., \& Padovani, P. 1988, ApJ, 340, L5.

Chen, K., Halpern, J.P., \& Filippenko, A.V. 1989, ApJ, 339, 742. 
Collin-Souffrin, S., \& Dumont, A. M. 1989, A\&A, 213, 29.

Cunningham, C. 1975, ApJ, 202, 788.

Dumont, A. M., \& Collin-Souffrin, S. 1990, A\&AS, 83, 71.

Lynden-Bell, D. 1969, Nature, 223, 690.

Pérez, E., et al. 1986, MNRAS, 230, 353.

Piccinoti, G., et al. 1982, ApJ, 253, 485.

Rees, M. 1966, Nature, 211, 468.

Rokaki, E., Boisson, C., \& Collin-Souffrin, S. 1992, A\&A, 253, 57.

Rokaki, E., Collin-Souffrin, S., \& Magnan, C. 1993, A\&A, 272, 8.

Rokaki, E., \& Boisson, C. 1996, in preparation.

Rokaki, E., Economou, F., \& Lawrence, A. 1996, in preparation.

Shields, G. A. 1977, Astrophys. Lett., 18, 119.

Vermeulen, R. C., \& Cohen, M. H. 1994, ApJ, 430, 467. 